\title{
The Dentistry Conference Houston
}

\author{
Ferdynand Barbasiewicz ${ }^{1 *}$ and Monika Michalska ${ }^{2}$ \\ ${ }^{1}$ Department of Dentistry, Non-invasive, molecular method of biocybernetic clavitherapy, Poland \\ ${ }^{2}$ Consultant and collaborator, Poland
}

*Corresponding author: Ferdynand Barbasiewicz, Department of Dentistry, Poland.

Received Date: January 19, 2021

Published Date: February 17, 2021

\section{Abstract}

Most early visual diagnostics of cranial nerves pathologies in the natural history of oral cavity and craniofacial disorders (demyelinating neuropathy, infections, and neuronal gliomatosis). Rapid restoration of immunity using the molecular method of biocybernetic clavitherapy by dr. Ferdynand Barbasiewicz.

\section{Clinical diagnostics of neuropathy}

Chronic infections with edema lead to activation of destructive psychosomatic mechanisms whereas painkillers administered over several days worsen the restricted circulation of blood within the infected venules. If the infection and swelling area involves the second or the third branch of the trigeminal nerve, enzyme metabolic function may be impaired at sub molecular level due to insufficient blood supply. This leads to the failure of aggregation of peripheral blood interferons acting as biocybernetic mediators responsible for subsequent aggregation of lipids, cytokines, endorphins, and other molecular complements making up the myelin sheath into the oligodendrocyte loss zone. As the result, the myelin sheath insulation of the trigeminal nerve fiber becomes deteriorated, leading to reduced nerve potentials in the decay latency period ,responsible for regulation of the enzyme metabolic function or the function of the pyramidal tract fiber dynamizing the skeletal muscles of the oral cavity and splachnocranium. Reduced blood supply is also observed within the sclerotome and periosteum of local dentition. Moreover, infections involving edemas disrupt the blood supply to the dermatome, connective tissue, neurotome, and myotome. Sentinel lymph nodes are also affected by inflammation. What happens when the infection involves the second or the third branch of the trigeminal nerve, with decay latency potentials being markedly reduced or even zeroed in EMG examinations? In such cases, pharmacotherapeutics listed above not only fail to treat, but instead exacerbate the infection as the result of e.g. venular ischemia being worsened. This may lead to accelerated, progressive infection-related pain reaction and pathological ischemic edema being expanded beyond the dentition level to effect sclerotomal and periosteal atrophization. After several (10+) days, hyperalgesicalgostic spots, slightly pink in color, sized 1 to $7 \mathrm{~mm}$, may develop following the infection crisis above the second or third trigeminal nerve fibers. This finding provides a diagnostic information on the loss of the first myelin sheath layer with partial discharge of body's nerve potentials with reduced decay latency levels. Progression of this skin symptom during persistent infection with continuous ischemia and edema will be associated with the loss of oligodendrocytes within the second, i.e. the last myelin sheath. This will lead to hypoesthetic, algodystrophic spot developing above the neuropathic site, with body's own nerve potentials being discharged into organic molecules while decay latency may reach the zero value in EMG examinations. Notably, neuropathy of nerve fibers which control the enzyme metabolic function of cell division at the sclerotome and periosteal level results in metabolic atrophism of dentition beyond the local level, resulting in teeth crumbling like moist brick below the freezing temperature. In this condition, extraction remains the only option.

\section{Management and Clinical Description of the Molecular Method of Biocybernetic Clavitherapy in Dentistry}

In case of an early-stage infection, e.g. that of a mouth ulcer in the vicinity of gums, take a sharp toothpick and press (strongly yet non-invasively) several times onto acupuncture site GRT-27 located on the main posterior regulating meridian, along thee median line below the turbinate bone and just above the red lip border. Each time, hold the pick for several seconds with one second intervals. 
Repeat 3-5 times a day. The procedure facilitates elimination of the mouth ulcer using patient's own selective antibodies. In the case of physical trauma to the gum and the periosteum involving slight of the tooth, Use two sharp toothpicks to immediately perform dermovisceral compression at the dermatome and connective tissue level above the periodontal lesion site; at the same time, use two toothpick held in the other hand to compress the extrameridian point PaM (see Atlas, p. 94) responsible for elimination of paradontosis). Hold for 3-5 seconds, repeat several times a day over 3-5 days. Initially, this will cause some pain; however, it would resolve within several days along with the infection and the swelling. For several days, patients should try to chew food using the other side of their mouth. The proposed stimulation method will result in very good blood supply for successful, drug-free elimination of post-traumatic symptoms with initial edema.

Similar symptoms facilitating the clinical diagnosis of neuropathy consist in the presence of demyelination plaque being preset within the brain, the motor neurons, and the peripheral nervous system. Notably, stress, frustration, and prolonged irritation disturb the function of thyroid and parathyroid glands; when disturbed, the glands shrink and secrete lower quantities of calcium and phosphorus into peripheral blood. - Stress-induced reduction in calcium and phosphorus ions being released from the parathyroids into peripheral blood is maintained is the main cause of reduced dietary intake of calcium and phosphorus upon digestion in the jejunum and ileum. This is the direct cause of reduced bone strength in some patients. Dietary supplements are ineffective in these cases.

\section{Clinical elimination of neuropathy}

Unpigmented, slightly pink-colored, algostic spot sized 1 to 3-7 $\mathrm{mm}$ observed within the maxillofacial area in the vicinity of the second or third branch of the trigeminal nerve or at other skin locations, either on the left or on the right, showing signs of hyperalgesia upon being impinged with a sharp toothpick is a manifestation of early-stage demyelinating neuropathy. It is a sign of the loss of the first myelin sheath layer, e.g. that of the second or the third branch of the trigeminal nerve, with partially reduced potentials being discharged into the organic molecules. In such cases, two sharp toothpicks should be use to compress the spots so as to stimulate increased blood supply with aggregation of interferons as biocybernetic mediators which, upon further stimulation, will trigger neurotransmitter-mediated accumulation of lipids, cytokines endorphins, and other hitherto unknown molecular endogenous factors so that the loss of oligodendrocytes forming the myelin sheath is reversed within several days. Following restoration of the immunity of the nerve fiber, own nerve potentials which regulate the enzyme metabolic function at submolecular level are restored to effective decay latency levels. Similar healing will also be observed within sclerotomal and periosteal cells in a relatively young person. On the other hand, white algo dystrophic plaque, hypoalgesic upon stimulation using two toothpicks or slight compression, develops as the result of ignorance and negligence on facial skin or in other locations, spots should be stimulated using two toothpicks until piercing pain, lasting a fraction of a second, is experienced by the patient to evidence forced blood supply that will result in neurotransmiter-mediated aggregation of interferons originating from nearby healthy cells. Other endogenous agents, including lipids, cytokines, endorphins, and hitherto unknown complements will be subsequently attracted to the hyperemic plaque. Following several days stimulation, oligodendrocyte cells comprising both layers of the myelin sheath will be restored and unimpaired neural transmission will resume for ongoing regeneration of sclerotomal and periodontal cells within permanent dentition (only in relatively young patients in good general health). Caution! Lack of sustained stimulation-related pain following some period of myelin sheath regeneration indicates a deficit of body's own interferons in the peripheral blood. In such case, stimulation of biologically active points responsible for increased endogenous production of interferons should be attempted. To this end, the following points should be simulated (appropriate locations to be found in the Atlas of Clavitherapy): Pm-62, p. 70; Wż-34, p. 84; Pm-54, p. 66; auricle, U-25 and 28 (growth factor to be compressed simultaneously using 2 toothpicks with feedback stimulation of umbilical cord rim line simultaneously compressed using 2 toothpicks) 3 times for several days until the pain resolves (do not puncture the navel; thermal stimulation using no-flame moxibustion allowed).

When no care is provided on time to infections involving edema, capillary veins become affected. At the early stage of infection, venular blood flow follows a reciprocal exchange pattern. In order to alleviate infection and edema surrounding a dental root, moderately, strong, non-invasive, perpendicular compression should be performed using the tip of a toothpick projected above the ischemically infected root. Depending on the extent of infection, the compression should last between 1 and 2-3 minutes until pain is resolved. The procedure may be performed by the patient themselves. Initially, pain will intensify. However, stimulation should loosen collagen fibers within the venules to restore the trace blood supply. Along with the inflowing blood, appropriate antibodies will be delivered for rapid alleviation of pain, edema, and infection and subsequent complete restoration of venular blood flow. As the result, the edema and infection can be resolved within about a quarter of an hour. Other stimulation spots include these at the inferior rim of the auricle, namely U-72.5 and 72.6, see Atlas p. 169. According to the Clavitherapy handbook, human auricle features a biologically active point U-55 (Atlas p. 169) which, when activated using an acupuncture needle, immediately triggers body's own endorphins and opioids to neutralize pain on the side of the planned major yet short-lasting dental procedure such as tooth extraction 
Most early diagnostics and efficient pathomorphological treatment of malignancies with straight-line metastatic pathways with liquidation of sentinel lymph node inflammation.

\section{Etiology}

For 70 thousand years, humans have been exposed to smoke generated by various materials subjected to combustion for different purposes. As shown by WHO-commissioned analyses carried out in Finland in years 2002-2004, cigarette smoke alone contains as much as 4500 toxic chemicals, including numerous carcinogens responsible for the development of various malignancies, not only those developing within the oral cavity. The increase in the number and concentration of airborne toxic chemicals due to all types of combustion and agricultural chemization processes is responsible for increased incidence of many diseases, particularly malignant neoplasms. At the early stage, malignancies with straight-line metastatic pathways can be diagnosed visually by several millimeterwide bands of lighter discoloration due to insufficient blood supply to the skin surrounding some pathological, brown nevi, papillomas, and melanocytic spots, as well as continuing along the straight lines of metastases between these lesions (see straight lines of tissue metastases, Atlas of Clavitherapy p. 19). Another diagnostic confirmation consists in no pain being experienced by patient upon malignant lesions being compressed using 2 toothpicks; this is sue to tight aggregation of cancer proteins leading to complete ischemia and paresis of neural cells. When immunity is impaired, excessive aggregation of cancer proteins occurs in the areas of malignantly transformed lesions due to long-term exposure to consumption of chemized foods, exposure to sunlight (particularly harmful due to the ozone hole and depletion of ozone layer at the altitude of about $30 \mathrm{~km}$ ), or the use of tanning salons - see photographs on Atlas pages 19,20 , and 21. Tight aggregation of cancer proteins delivered with peripheral blood successively extends the ischemic zone, initially within the dermatome and connective tissue as well as deeper endogenous cells and tissue levels in more immunocompromised patients. Cancer proteins and various types of markers may paralyze neurophysiological function of neurons within the brain and peripheral nervous system. In immunocompromised patients, the process leads to the development of gliomas within brain and the peripheral system; with time, the developing pathologies may become untreatable by chemotherapy due to the lack of circulation. As we know, chemotherapeutics are delivered to the target site with blood. In an environment encompassing the metastasis pathways, for example in the trigeminal nerve region, ischemia leads to postinfective neuralgia and paralysis of nerve signals that control cell functions at different endogenous levels, with simultaneous inflammation of sentinel lymph nodes.

Clinical elimination of aggregation of cancer proteins by means of non-invasive vibration using two 5-mm clavicles/tooth picks, for forced delivery of antibodies with qualitatively restored own blood to the earliest signs of aggregation of cancer proteins including metastatic lines, starting from careful generation of hyperemia around the pathological lesions and metastatic lines and stimulation of biologically active points to eliminate inflammation of sentinel lymph nodes as described in Clavitherapy handbook, page 261, algorithm 1.21, with topographic references to the Atlas. Killers, i.e. body's own selective antibodies in delivered in blood following full restoration of immunity using the molecular method of biocyberneticclavitherapy, starting from the aforementioned disorders and pathologies (see Atlas of Clavitherapy, pp. 19, 20, and 21), with supportive exercises (see Atlas, p. 172) , for restoration of full blood supply, own nerve potentials, and normal neurophysiology of affected neurons, including restoration of enzyme-metabolic function in other cells and tissues at all endogenous levels, cell divisions, and production of 3 million of different types of molecular factors, 6 millions of neurochemical transmitters and 3 millions of different submolecular transmitters for effective restoration of blood immunity. Of course, this process is most efficient in relatively young and less immunocompromised patients receiving no drugs of any type (it should be kept in mind that sites of tight aggregation of cancer proteins and markers, including malignant melanoma, are devoid of blood supply and thus can't be reached by chemotherapeutic agents). There is no need for Co-60 irradiations, blood transfusions, or any supplements which have never been examined at the molecular level. Blood pH should be monitored; if the blood is too acidic, e.g. $\mathrm{pH}=5, \mathrm{PaM}-138$ should be stimulated until resolution of pain 3 times a day over several days (see Atlas of Clavitherapy, pp. 118 and 122), until more alkaline pH of 6-7 is achieved. Acidity may be regulated using aqueous solution of baking soda according to the universally known method.

\section{A Method to Restore the Immunity within 7-12 Days Depending on Patient's Age and Type of Local or Systemic Infecton Risk in Relatively Young Subjects.}

Biologically active sites for non-invasive stimulation according to the new edition schedule with topographical references to the clavitherapy atlas:

- PaM-3: relaxation of cortical stress to eliminate burning and pain; Atlas pp. 26 and 35;

- $\quad$ GRT-15 and 16: relaxation of central stress to eliminate burning and pain; Atlas p. 36;

- $\quad$ GRP-22: relaxation of thyroid; Atlas p. 35;

- GRT-14: elimination of neuromuscular trauma, burning, and pain, cryogenic site; Atlas p. 36

- GRP-12: elimination of vegetative gastrointestinal neurosis; Atlas p. 92;

- $\quad$ Pm-13, bilateral; detoxification of lugs; Atlas, p. 66;

- Elimination of anterior and posterior pituitary gland disorders; Clavitherapy handbook p. 268, algorithm5.1; 
- $\quad$ Pm-43, bilateral: cortisone activation; Atlas p. 66;

- $\quad$ Pm-16 and 17, bilateral: cryogenic, Atlas p. 66;

- Pm-47, bilateral: detoxification of liver, Atlas p. 66;

- $\quad \mathrm{W}-13$ and 14, bilateral: regulation of transaminases and eradication of all parasites; Atlas p. 88;

- $\quad$ GRP-19 and 20, reconstruction of T4 and T8 lymphocytes, Atlas p. 92;

- $\quad$ GRT-2 and PaM-41, bilateral: elimination of anemia; Atlas pp. 90 and 100;

- $\quad$ ST-13, bilateral: heavy metals detoxification; Atlas p. 60

- ST-10, bilateral: immunity in elimination of allergies; Atlas p. 58;

- $\quad \dot{Z}-36$, bilateral: the great healer; Atlas p. 56;

- Wż-37 and 39, bilateral: cryogenic immunity, Atlas p. 84;

- ST-6, bilateral: immunity, supportive in elimination of atherosclerosis; Atlas p. 58;

- U-25 and 28: growth factor with feedback stimulation of umbilical cord rim line for stem call reconstruction; Atlas pp. 35 and 168.

- U-82 and 83, bilateral: cryogenic immunity, Atlas p. 169;

- elimination of needle pain by means of two sharp toothpicks stimulating the areolae. Caution! Stimulation of this zone is forbidden in pregnant or breastfeeding women due to the risk of lactation being disrupted through stimulation of site 0-1. Caution! If patient fails or collapses during the procedure, no ambulance defibrillation service or chest massage are required. Patient should be quickly placed in an appropriate position and
GRT-26 stimulation should be undertaken to resuscitate or reanimate the patient as shown in the photograph (see Atlas p. 15). Resuscitation and reanimation are best achieved using clavitherapy. When aiming to restore immunity, one should first eliminate the aforementioned neuropathy and neuronal paresis caused by cancer proteins along the metastatic lines at the pathomorphological level; sentinel lymph node inflammation should also be eliminated - see Clavitherapy handbook, p. 261, and Atlas references cited therein. When restoring immunity, clavitherapy exercises to increase blood supply to all cells and tissues should be practiced at the endogenous level from the very start of the proceedings - see Atlas p. 172. - More clinical examples are included in the Clavitherapy handbook (in Polish); two copies of the book will be presented to the Congress board.

P.S. Due to the significant expenses borne in the course of pro bono clinical studies on the molecular method of biocyberneticclavitherapy as well as in relation to the writing and publishing of the three-volume Clavitherapy handbook and translating of numerous publications into English we are asking that the Author and his Collaborator are exempted from registration fees as well as travel and accommodation costs. We are also asking that a number of patients with the aforementioned diagnoses are available at the workshop so that their neuropathy and cancer protein-related paresis may be eliminated in public to demonstrate the efficiency of the method. Attendants will be able to document the pathologies and the treatment procedure by video recording.

\section{Acknowledgement}

None.

\section{Conflict of Interest}

No Conflict of Interest. 